MATHEMATICS OF COMPUTATION

Volume 66, Number 217, January 1997, Pages 1-14

S $0025-5718(97) 00785-0$

\title{
LOCKING-FREE FINITE ELEMENT METHODS FOR SHELLS
}

\author{
DOUGLAS N. ARNOLD AND FRANCO BREZZI
}

\begin{abstract}
We propose a new family of finite element methods for the Naghdi shell model, one method associated with each nonnegative integer $k$. The methods are based on a nonstandard mixed formulation, and the $k$ th method employs triangular Lagrange finite elements of degree $k+2$ augmented by bubble functions of degree $k+3$ for both the displacement and rotation variables, and discontinuous piecewise polynomials of degree $k$ for the shear and membrane stresses. This method can be implemented in terms of the displacement and rotation variables alone, as the minimization of an altered energy functional over the space mentioned. The alteration consists of the introduction of a weighted local projection into part, but not all, of the shear and membrane energy terms of the usual Naghdi energy. The relative error in the method, measured in a norm which combines the $H^{1}$ norm of the displacement and rotation fields and an appropriate norm of the shear and membrane stress fields, converges to zero with order $k+1$ uniformly with respect to the shell thickness for smooth solutions, at least under the assumption that certain geometrical coefficients in the Nagdhi model are replaced by piecewise constants.
\end{abstract}

\section{INTRODUCTION}

A number of important variational problems of mechanics involve an internal energy functional of the form $E_{1}+\epsilon^{-2} E_{2}$ with $\epsilon$ a parameter that may become small. For example, the displacement energy of an isotropic, linearly elastic material has this form, where $E_{1}$ and $E_{2}$ give the contributions to the energy due to the deviatoric and dilational strains, respectively, and $\epsilon=\sqrt{1-2 \nu}$ with $\nu$ the Poisson ratio (so $\epsilon$ is small for nearly incompressible materials). A second example is provided by the Reissner-Mindlin model of plate bending. In this case, $E_{1}$ represents the elastic energy due to bending and $E_{2}$ that due to transverse shear. The small parameter $\epsilon$ is the plate thickness. A third example is given by the Koiter shell model, with the decomposition this time corresponding to energy due to bending and that due to membrane stresses, and $\epsilon$ again representing the thickness. The case considered in this paper is the shell model of Naghdi [14], [15]. Here, $E_{1}$ again represents the elastic energy due to bending while $E_{2}$ is now the sum of the contributions to the energy due to transverse shear and that due to membrane stresses. More information on these models can be found in many texts, e.g., [5], [12], [18].

Received by the editor December 2, 1993 and, in revised form, April 3, 1995 and November 13, 1995.

1991 Mathematics Subject Classification. Primary 65N30, 73K15, 73V05.

Key words and phrases. Shell, locking, finite element.

The work of the first author was supported by National Science Foundation grants DMS9205300 and DMS-9500672. The work of the second author was partially supported by the HCM Program on Shells, contract number ERBCHRXCT 940536.

(C)1997 American Mathematical Society 
When the finite element method is used to discretize a problem of this sort, that is, when the total energy - which is the internal energy plus that due to the loading - is minimized over a finite element space, the convergence of the method will typically deteriorate as $\epsilon$ becomes small. In fact, for many standard choices of finite element spaces there is no convergence uniform in $\epsilon$ as the mesh is refined (although it is certainly true that for any fixed $\epsilon$ the discrete solution converges to the exact solution). In practice, one sees a large discretization error when $\epsilon$ is small, even for quite refined meshes. Indeed, in many practical computations it may well be impossible to achieve an acceptable level of error using standard finite elements. This phenomenon of growing discretization error as $\epsilon$ decreases is referred to as locking. For the problem of nearly incompressible elasticity, the term Poisson locking is used. For the Reissner-Mindlin model, one speaks of shear locking, and for the Koiter shell model, membrane locking. In the case of the Naghdi shell model studied in this paper both shear and membrane locking enter.

The usual approaches to circumventing locking are the use of special finite element spaces and/or the alteration of the energy form. These techniques are often equivalent to the use of stable elements for a mixed variational reformulation of the problem. There is a considerable literature devoted to the development and mathematical justification of locking-free finite elements. For Poisson locking, which is closely related to the limiting Stokes problem, many locking-free methods are known. Cf. [11] or [7]. For the Reissner-Mindlin plate, several methods have been proven to be free of locking in recent years [2], [7, Ch. 7]. However, as far as we know, very little mathematical analysis has been done for locking in shell problems. In the pioneering paper [16] some methods are analyzed in the special case of bending-dominated cylindrical shells. The results are illuminating, but also quite discouraging; in particular, for low-order methods convergence is shown only for tensor product meshes. In spite of its importance, the problem of finding numerical methods for shells that can be mathematically proven to be locking-free is still wide open.

In this paper we present a sequence of mixed finite element methods for the Naghdi shell, one for each positive integer order, which we conjecture to be lockingfree in that the relative error in the numerical solution tends to zero with an order limited only by the approximation properties of the finite elements and the smoothness of the solution, but which is uniform with respect to the shell thickness. While we have not proved this in general, we prove it to be the case under the assumption that the coefficients which enter the internal energy expression are piecewise constant functions. This assumption, which is very restrictive, is motivated by our technique of analysis. We do not believe that it is necessary. The exact statement of the result is given in $\S 4$, where its interpretation is discussed more fully. In particular the norm we use to measure the approximation error couples the primitive variables of the Naghdi model (displacement and rotation) with auxilliary stress variables. It may happen that the relative error in the primitive variables alone does not tend to zero with optimal order, uniformly in the plate thickness. As discussed in that section, in the case of bending-dominated shell problems, the case for which locking is generally considered to be most troublesome, there is uniform convergence of the relative error in the primitive variables. However, this may not occur for membrane-dominated problems, and so our methods may not be appropriate for such problems. 
The mixed formulation we introduce is not quite the standard one. It is based on a splitting of the shear and membrane energy terms so that part of each of these terms is included with the bending energy term before passing to the mixed formulation. It turns out that this minor alteration to the standard mixed formulation renders the development of stable mixed elements much simpler. This idea, which, in the limiting case of zero plate thickness, reduces to the use of an augmented Lagrangian, has appeared before in many contexts. For scalar second-order elliptic problems it can be found in [8]. We applied it to the Reissner-Mindlin plate in [1] as did Zhou in [17]. For an application to an orthotropic heat flow problem, see [4]. In the context of shells, this idea is mentioned in [16]; cf. (4.10) and Remark 4.1 in that paper.

Of course there is a vast literature on the numerical approximation of shells, and many works are concerned, either explicitly or implicitly, with the development of accurate, locking-free methods, even if no mathematical theory is available. Rather than selectively reference that literature, we refer the reader to the books [5], [12], [18].

The organization of the paper is as follows. In the next section we present the Naghdi model and the mixed formulation for it. In $\S 3$ a simple abstract framework is presented in preparation for the analysis of $\S 4$. In that section, the finite element methods are presented and the uniform convergence in $\epsilon$ is proved.

\section{The NAghd Shell MOdel AND A MiXed Formulation}

We employ the convention that Greek indices range over 1 and 2 and Latin indices over 1, 2, and 3. Products containing repeated indices (which will always occur as a subscript and a superscript) are summed. Thus, for example, $b_{i}^{\alpha}=a^{\alpha \beta} b_{\beta i}$ means that $b_{i}^{\alpha}=a^{\alpha 1} b_{1 i}+a^{\alpha 2} b_{2 i}$ for $i=1,2,3$ and $\alpha=1,2$. We use overarrows to indicate 3 -vectors, undertildes to indicate 2 -vectors, and double undertildes to indicate $2 \times 2$ symmetric tensors. Thus $\vec{v}=\left(v_{i}\right), \underset{\sim}{v}=\left(v_{\alpha}\right)$, and $\underset{\approx}{\lambda}=\left(\lambda_{\alpha \beta}\right)$ with $\lambda_{\alpha \beta}=\lambda_{\beta \alpha}$.

For simplicity we suppose that the middle surface of the shell can be represented by a single chart. Let $\Omega$ be a bounded open set in $\mathbb{R}^{2}$ with closure $\bar{\Omega}$ and let $\vec{r}$ be a smooth one-to-one mapping of $\bar{\Omega}$ into $\mathbb{R}^{3}$, for which the vectors

$$
\overrightarrow{a_{1}}=\frac{\partial \vec{r}}{\partial x^{1}}, \quad \overrightarrow{a_{2}}=\frac{\partial \vec{r}}{\partial x^{2}}
$$

are linearly independent at each point of $\bar{\Omega}$. Then for each $\underset{\sim}{x} \in \Omega$, the vectors $\overrightarrow{a_{1}}(\underset{\sim}{x})$ and $\overrightarrow{a_{2}}(\underset{\sim}{x})$ define a basis for the tangent space to the midsurface $\bar{S}=\vec{r}(\bar{\Omega})$ at the point $\underset{r}{\vec{\sim}} \underset{\sim}{x})$. We also set

$$
\overrightarrow{a_{3}}=\frac{\overrightarrow{a_{1}} \times \overrightarrow{a_{2}}}{\left|\overrightarrow{a_{1}} \times \overrightarrow{a_{2}}\right|},
$$

which is a unit normal vector at $\vec{r}(\underset{\sim}{x})$, so that the vectors $\overrightarrow{a_{1}} \underset{\sim}{(x)}, \overrightarrow{a_{2}} \underset{\sim}{(x)}, \underset{a_{3}}{(x)}$ form a basis for $\mathbb{R}^{3}$. We let $t$ denote the shell thickness, so that the shell occupies the region

$$
\left.\left\{\vec{r}(\underset{\sim}{x})+x^{3} \overrightarrow{a_{3}} \underset{\sim}{x}\right) \mid \underset{\sim}{x} \in \Omega,-t / 2<x^{3}<t / 2\right\} \subset \mathbb{R}^{3} .
$$


The first and second fundamental forms of the midsurface are defined by the $2 \times 2$-matrix-valued functions given by

$$
a_{\alpha \beta}=\overrightarrow{a_{\alpha}} \cdot \overrightarrow{a_{\beta}}, \quad b_{\alpha \beta}=\overrightarrow{a_{3}} \cdot \frac{\partial^{2} \vec{r}}{\partial x^{\alpha} \partial x^{\beta}}=\overrightarrow{a_{3}} \cdot \frac{\partial \overrightarrow{a_{\alpha}}}{\partial x^{\beta}}=-\overrightarrow{a_{\alpha}} \cdot \frac{\partial \overrightarrow{a_{3}}}{\partial x^{\beta}}
$$

The last equality follows from the orthogonality of $\overrightarrow{a_{\alpha}}$ and $\overrightarrow{a_{3}}$. Note that both fundamental forms are symmetric in $\alpha$ and $\beta$. We also define the inverse matrix to $\left(a_{\alpha \beta}\right)$ denoting its components by $a^{\alpha \beta}$. Setting $\overrightarrow{a^{\alpha}}=a^{\alpha \beta} \overrightarrow{a_{\beta}}$, we have $a^{\alpha \beta}=\overrightarrow{a^{\alpha}} \cdot \overrightarrow{a^{\beta}}$. Multiplication by $a^{\alpha \beta}$ raises indices on other tensors as well, so $a^{\alpha \beta} b_{\beta \gamma}=b_{\gamma}^{\alpha}$. Finally, we let $a$ denote the determinant of the matrix $\left(a_{\alpha \beta}\right)$, a never-vanishing function on $\bar{\Omega}$, so that if $f: S \rightarrow \mathbb{R}$ is any function, then $\int_{S} f=\int_{\Omega} f(\vec{r}(\underset{\sim}{\vec{r}})) \sqrt{\bar{a}} d x$.

For an arbitrary displacement field $\vec{u}=\left(u_{i}\right)$ and rotation field $\underset{\sim}{\theta}=\left(\theta_{\alpha}\right)$ with $u_{i}, \theta_{\alpha} \in H^{1}(\Omega)$, define the change of curvature tensor $\underset{\approx}{\Upsilon}$, the transverse shear strain tensor $\underset{\sim}{\Phi}$, and the membrane strain tensor $\underset{\approx}{\Lambda}$ by

(1) $\Upsilon_{\alpha \beta}(\vec{u}, \underset{\sim}{\theta})=\frac{1}{2}\left[\theta_{\alpha \mid \beta}+\theta_{\beta \mid \alpha}-b_{\alpha}^{\gamma}\left(u_{\gamma \mid \beta}-b_{\gamma \beta} u_{3}\right)-b_{\beta}^{\gamma}\left(u_{\gamma \mid \alpha}-b_{\gamma \alpha} u_{3}\right)\right]$

$$
\begin{aligned}
=\frac{1}{2} & {\left[\theta_{\alpha, \beta}+\theta_{\beta, \alpha}-b_{\alpha}^{\gamma}\left(u_{\gamma, \beta}-\Gamma_{\gamma \beta}^{\delta} u_{\delta}\right)\right.} \\
& \left.\quad-b_{\beta}^{\gamma}\left(u_{\gamma, \alpha}-\Gamma_{\gamma \alpha}^{\delta} u_{\delta}\right)+b_{\alpha}^{\gamma} b_{\gamma \beta} u_{3}\right]-\Gamma_{\alpha \beta}^{\delta} \theta_{\delta},
\end{aligned}
$$

(2) $\Phi_{\alpha}(\vec{u}, \underset{\sim}{\theta})=u_{3 \mid \alpha}+b_{\alpha}^{\gamma} u_{\gamma}+\theta_{\alpha}=u_{3, \alpha}+b_{\alpha}^{\gamma} u_{\gamma}+\theta_{\alpha}$,

$$
\Lambda_{\alpha \beta}(\vec{u})=\frac{1}{2}\left(u_{\alpha \mid \beta}+u_{\beta \mid \alpha}\right)-b_{\alpha \beta} u_{3}=\frac{1}{2}\left(u_{\alpha, \beta}+u_{\beta, \alpha}\right)-\Gamma_{\alpha \beta}^{\delta} u_{\delta}-b_{\alpha \beta} u_{3},
$$

where the Christoffel symbol $\Gamma_{\alpha \beta}^{\delta}:=\overrightarrow{a^{\delta}} \cdot \partial \overrightarrow{a_{\beta}} / \partial x^{\alpha}$.

Set

$$
a^{\alpha \beta \gamma \delta}=\frac{E}{1-\nu^{2}}\left(a^{\alpha \gamma} a^{\beta \delta}+\nu a^{\alpha \beta} a^{\gamma \delta}\right)
$$

with $E>0$ and $\nu \in(0,1 / 2)$ denoting the Young's modulus and Poisson ratio of the material. Let $\Gamma_{d}$ denote a nonempty subset of $\partial \Omega$ and set

$$
H_{\Gamma_{d}}^{1}=\left\{v \in H^{1}(\Omega)|v|_{\Gamma_{d}}=0\right\}, \quad V=\left\{(\vec{v}, \underset{\sim}{\psi}) \mid v_{i}, \psi_{\alpha} \in H_{\Gamma_{d}}^{1}\right\}
$$

The norm in $V$ is given by $\|\vec{u}, \underset{\sim}{\psi}\|_{V}^{2}=\|\vec{v}\|_{1}^{2}+\|\underset{\sim}{\psi}\|_{1}^{2}$. The Naghdi model determines $(\vec{u}, \underset{\sim}{\theta})$ as the minimizer over $V$ of the energy functional

$$
\begin{aligned}
E(\vec{v}, \underset{\sim}{\psi})= & E^{B}+E^{S}+E^{M}+E^{L} \\
:= & \frac{t^{3}}{2} \int_{\Omega} \frac{a^{\alpha \beta \gamma \delta}}{12} \Upsilon_{\alpha \beta}(\vec{v}, \underset{\sim}{\psi}) \Upsilon_{\gamma \delta}(\vec{v}, \underset{\sim}{\psi}) \sqrt{a} d \underset{\sim}{\vec{\sim}} \\
& +\frac{t}{2} \int_{\Omega} a^{\alpha \beta} \frac{E}{2(1+\nu)} \Phi_{\alpha}(\vec{v}, \underset{\sim}{\psi}) \Phi_{\beta}(\vec{v}, \underset{\sim}{\psi}) \sqrt{a} d x \\
& +\frac{t}{2} \int_{\Omega} a^{\alpha \beta \gamma \delta} \Lambda_{\alpha \beta}(\vec{v}) \Lambda_{\gamma \delta}(\vec{v}) \sqrt{a} d \underset{\sim}{x}-t^{3} \int_{\Omega} f^{i} v_{i} \sqrt{a} d x .
\end{aligned}
$$


Here, $t^{3} f^{i} \overrightarrow{a_{i}}$ denotes the resultant of the applied forces on the middle surface. Equivalently, $(\vec{u}, \underset{\sim}{\theta}) \in V$ is determined by the weak equations

$$
\begin{gathered}
\int_{\Omega} \frac{a^{\alpha \beta \gamma \delta}}{12} \Upsilon_{\alpha \beta}(\vec{u}, \underset{\sim}{\theta}) \Upsilon_{\gamma \delta}(\vec{v}, \underset{\sim}{\psi}) \sqrt{a} d \underset{\sim}{x}+t^{-2} \int_{\Omega} a^{\alpha \beta} \frac{E}{2(1+\nu)} \Phi_{\alpha}(\vec{u}, \underset{\sim}{\theta}) \Phi_{\beta}(\vec{v}, \underset{\sim}{\underset{\sim}{\psi}}) \sqrt{a} d \underset{\sim}{\sim} \\
+t^{-2} \int_{\Omega} a^{\alpha \beta \gamma \delta} \Lambda_{\alpha \beta}(\vec{u}) \Lambda_{\gamma \delta}(\vec{v}) \sqrt{a} d \underset{\sim}{x}=\int_{\Omega} f^{i} u_{i} \sqrt{a} d \underset{\sim}{\sim} \quad \text { for all }(\vec{v}, \underset{\sim}{\psi}) \in V .
\end{gathered}
$$

To define a mixed formulation, we set

$$
\begin{gathered}
\phi_{\alpha}=\left(t^{-2}-c_{0}\right) \frac{E}{2(1+\nu)} \Phi_{\alpha}(\underset{\sim}{\vec{u}} \underset{\sim}{\theta}), \\
\lambda_{\gamma \delta}=\left(t^{-2}-c_{0}\right) \frac{E}{1-\nu^{2}}\left[\Lambda_{\gamma \delta}(\vec{u})+\nu a_{\gamma \delta} a^{\alpha \beta} \Lambda_{\alpha \beta}(\vec{u})\right]
\end{gathered}
$$

and seek pairs $(\vec{u}, \underset{\sim}{\theta}) \in V$ and $\underset{\sim}{\phi} \underset{\sim}{\lambda}) \in W:=\left\{(\underset{\sim}{\eta}, \underset{\sim}{\chi}) \mid \eta_{\alpha}, \chi_{\alpha \beta} \in L^{2}(\Omega)\right\}$ such that

$$
\begin{aligned}
& A(\vec{u}, \underset{\sim}{\theta} ; \vec{v}, \underset{\sim}{\psi})+B(\vec{v}, \underset{\sim}{\psi} ; \underset{\sim}{\phi} \underset{\sim}{\lambda})=F(\vec{v}) \quad \text { for all }(\vec{v}, \underset{\sim}{\psi}) \in V, \\
& B(\vec{u}, \underset{\sim}{\theta} ; \underset{\sim}{\eta} \underset{\sim}{\chi})-\frac{t^{2}}{1-c_{0} t^{2}} C(\underset{\sim}{\phi}, \underset{\approx}{\lambda} \underset{\sim}{\eta} \underset{\sim}{\chi})=0 \quad \text { for all }(\underset{\sim}{\eta}, \underset{\approx}{\chi}) \in W .
\end{aligned}
$$

Here,

$$
\begin{aligned}
& A(\vec{u}, \underset{\sim}{\theta} ; \vec{v}, \underset{\sim}{\psi})=\int_{\Omega} \frac{a^{\alpha \beta \gamma \delta}}{12} \Upsilon_{\alpha \beta}(\vec{u}, \underset{\sim}{\theta}) \Upsilon_{\gamma \delta}(\vec{v}, \underset{\sim}{\psi}) \sqrt{a} d \underset{\sim}{x} \\
& +c_{0} \int_{\Omega} a^{\alpha \beta} \frac{E}{2(1+\nu)} \Phi_{\alpha}(\vec{u}, \underset{\sim}{\theta}) \Phi_{\beta}(\vec{v}, \underset{\sim}{\psi}) \sqrt{a} d \underset{\sim}{\sim} \\
& +c_{0} \int_{\Omega} a^{\alpha \beta \gamma \delta} \Lambda_{\alpha \beta}(\vec{u}) \Lambda_{\gamma \delta}(\vec{v}) \sqrt{a} d x, \\
& B(\vec{v}, \underset{\sim}{\psi} ; \underset{\sim}{\eta} \underset{\sim}{\chi})=\int_{\Omega} a^{\alpha \beta} \Phi_{\alpha}(\vec{v}, \underset{\sim}{\psi}) \eta_{\beta} \sqrt{a} d \underset{\sim}{x}+\int_{\Omega} a^{\alpha \beta \gamma \delta} \Lambda_{\alpha \beta}(\vec{v}) \chi_{\gamma \delta} \sqrt{a} d \underset{\sim}{x}, \\
& C(\underset{\sim}{\phi} \underset{\approx}{\lambda} ; \underset{\sim}{\eta} \underset{\approx}{\chi})=\int_{\Omega} a^{\alpha \beta} \frac{2(1+\nu)}{E} \phi_{\alpha} \eta_{\beta} \sqrt{a} d x+\int_{\Omega} a^{\alpha \gamma} a^{\beta \delta} \lambda_{\alpha \beta} \chi_{\gamma \delta} \sqrt{a} d x, \\
& F(\vec{v})=\int_{\Omega} f^{i} u_{i} \sqrt{a} d x
\end{aligned}
$$

Aside from the factor of $t^{-2}-c_{0}, \underset{\sim}{\phi}$ and $\underset{\approx}{\lambda}$ represent the transverse shear stresses and membrane stresses, respectively (note that $a^{\alpha \gamma} a^{\beta \delta} \lambda_{\alpha \beta}=\left(t^{-2}-c_{0}\right) a^{\alpha \beta \gamma \delta} \Lambda_{\alpha \beta}(\vec{u})$ ). The quantity $c_{0}$, which has the units of reciprocal of the square of length, is an arbitrary positive constant, independent of $t$. It may be chosen, for example, to be the reciprocal of the area of the middle surface of the plate. Different values could be taken for the two separate occurrences in equation (6). By insisting that $c_{0}$ be positive, rather than making the more usual choice $c_{0}=0$, we obtain the following result, which greatly simplifies the development of stable mixed finite elements. For a proof see [6].

Lemma 1. The bilinear form $A$ is an inner product on $V$ and the corresponding norm is equivalent to the $H^{1}$ norm. 


\section{An abstract stability Result}

In this section, in which the notation is independent of the others, we let $V$ and $W$ denote Hilbert spaces, and suppose that we are given three bounded bilinear forms

$$
A: V \times V \rightarrow \mathbb{R}, \quad B: V \times W \rightarrow \mathbb{R}, \quad C: W \times W \rightarrow \mathbb{R},
$$

a bounded linear form $F: V \rightarrow \mathbb{R}$, and a parameter $\epsilon \in(0,1)$. We consider the abstract problem:

Problem $A^{\epsilon}$. Find $u \in V, p \in W$ such that

$$
\begin{array}{ll}
A(u, v)+B(v, p)=F(v) & \text { for all } v \in V, \\
B(u, q)-\epsilon^{2} C(p, q)=0 & \text { for all } w \in W .
\end{array}
$$

We shall denote the bounds on the bilinear forms by $\|A\|,\|B\|$, and $\|C\|$. Moreover, we shall assume that $A$ and $C$ are positive definite, so there exist positive constants $c_{1}$ and $c_{2}$ for which $A(v, v) \geq c_{1}\|v\|_{V}^{2}$ and $C(w, w) \geq c_{2}\|w\|_{W}^{2}$ for all $v \in V$ and $w \in W$. We define a seminorm on $W$ by

$$
\|q\|=\sup _{0 \neq v \in V} \frac{B(v, q)}{\|v\|_{V}} \quad \text { for all } q \in W
$$

so that $\|q\|\|\leq\| B\|\| q \|_{W}$. The following theorem follows easily from the LaxMilgram lemma applied to the positive definite form

$$
((u, p),(v, q)) \mapsto A(u, v)+B(v, p)-B(u, q)+\epsilon^{2} C(p, q),
$$

the obvious choice of test functions, and the definition of the seminorm.

Theorem 2. There exists a unique solution $u, p$ to Problem $A^{\epsilon}$. Moreover, there is a constant $C$ depending only on $\|A\|,\|C\|, c_{1}$ and $c_{2}$, for which

$$
\|u\|_{V}+\|p\|\|+\epsilon\| p\left\|_{W} \leq C\right\| F \|_{V^{*}} .
$$

Now let $V_{h} \subset V$ and $W_{h} \subset W$ denote finite-dimensional subspaces, and consider the discrete problem:

Problem $A_{h}^{\epsilon}$. Find $u_{h} \in V_{h}, p_{h} \in W_{h}$ such that

$$
\begin{array}{ll}
A\left(u_{h}, v\right)+B\left(v, p_{h}\right)=F(v) & \text { for all } v \in V_{h}, \\
B\left(u_{h}, q\right)-\epsilon^{2} C\left(p_{h}, q\right)=0 & \text { for all } w \in W_{h} .
\end{array}
$$

Theorem 3. There exists a unique solution $u_{h}, p_{h}$ to Problem $A_{h}^{\epsilon}$. Moreover, there is a constant $C$ depending only on $\|A\|,\|B\|,\|C\|, c_{1}$ and $c_{2}$, for which

$$
\left\|u-u_{h}\right\|_{V}+\epsilon\left\|p-p_{h}\right\|_{W} \leq C \inf _{\substack{v \in V_{h} \\ q \in W_{h}}}\left(\epsilon^{-1}\|u-v\|_{V}+\|p-q\|+\epsilon\|p-q\|_{W}\right) .
$$

Proof. Existence and uniqueness is again a direct consequence of the Lax-Milgram lemma. To get the estimate, we note that for any $u^{*} \in V_{h}$ and $p^{*} \in W_{h}$ we have

$$
\begin{aligned}
& \begin{array}{l}
A\left(u_{h}-u^{*}, v\right)+B\left(v, p_{h}-p^{*}\right) \\
\quad=A\left(u-u^{*}, v\right)+B\left(v, p-p^{*}\right) \quad \text { for all } v \in V_{h}, \\
\quad B\left(u_{h}-u^{*}, q\right)-\epsilon^{2} C\left(p_{h}-p^{*}, q\right) \\
\quad=B\left(u-u^{*}, q\right)-\epsilon^{2} C\left(p-p^{*}, q\right) \quad \text { for all } q \in W_{h} .
\end{array}
\end{aligned}
$$


Choosing $v=u_{h}-u^{*}, q=p_{h}-p^{*}$ and subtracting (14) from (13), we get

$$
\begin{aligned}
& c_{1}\left\|u_{h}-u^{*}\right\|_{V}^{2}+c_{2} \epsilon^{2}\left\|p_{h}-p^{*}\right\|_{W}^{2} \\
& \quad \leq\|A\|\left\|u-u^{*}\right\|_{V}\left\|u_{h}-u^{*}\right\|_{V}+\left\|u_{h}-u^{*}\right\|_{V}\left\|p-p^{*}\right\| \| \\
& \quad+\|B\|\left\|u-u^{*}\right\|_{V}\left\|p_{h}-p^{*}\right\|_{W}+\epsilon^{2}\|C\|\left\|p-p^{*}\right\|_{W}\left\|p_{h}-p^{*}\right\|_{W} .
\end{aligned}
$$

We easily deduce that

$$
\left\|u_{h}-u^{*}\right\|_{V}+\epsilon\left\|p_{h}-p^{*}\right\|_{W} \leq C\left(\epsilon^{-1}\left\|u-u^{*}\right\|_{V}+\left\|p-p^{*}\right\|+\epsilon\left\|p-p^{*}\right\|_{W}\right),
$$

and then the desired estimate follows from the triangle inequality.

Notice that the estimates provided for $u-u_{h}$ and $p-p_{h}$ in Theorem 3 are not uniform in $\epsilon$. To obtain uniform estimates, we need a stability hypothesis. Namely, we shall assume that there exists $\gamma_{h}>0$ for which

$$
\sup _{0 \neq v \in V_{h}} \frac{B(v, q)}{\|v\|_{V}} \geq \gamma_{h}\|q\| \| \quad \text { for all } q \in W_{h} .
$$

We then obtain a quasi-optimal estimate uniformly in $\epsilon$.

Theorem 4. There exists a constant $C$ depending only on $\|A\|,\|C\|, c_{1}, c_{2}$, and $\gamma_{h}$, for which

$$
\left\|u-u_{h}\right\|_{V}+\left\|p-p_{h}\right\| \mid+\epsilon\left\|p-p_{h}\right\|_{W} \leq C \inf _{\substack{v \in V_{h} \\ q \in W_{h}}}\left(\|u-v\|_{V}+\|p-q\|+\epsilon\|p-q\|_{W}\right) .
$$

Proof. With the same choice of test functions as in the proof of the previous theorem, but treating the term $B\left(u-u^{*}, p_{h}-p^{*}\right)$ differently, we get

$$
\begin{aligned}
c_{1}\left\|u_{h}-u^{*}\right\|_{V}^{2}+c_{2} \epsilon^{2}\left\|p_{h}-p^{*}\right\|_{W}^{2} & \\
\leq & \|A\|\left\|u-u^{*}\right\|_{V}\left\|u_{h}-u^{*}\right\|_{V}+\left\|u_{h}-u^{*}\right\|_{V}\left\|p-p^{*}\right\| \\
& +\left\|u-u^{*}\right\|_{V}\left\|p_{h}-p^{*}\right\|+\epsilon^{2}\|C\|\left\|p-p^{*}\right\|_{W}\left\|p_{h}-p^{*}\right\|_{W} .
\end{aligned}
$$

Further, from (13) and (16) we have

$$
\gamma_{h}\left\|p_{h}-p^{*}\right\| \leq\|A\|\left\|u_{h}-u^{*}\right\|_{V}+\|A\|\left\|u-u^{*}\right\|_{V}+\left\|p-p^{*} \mid\right\|,
$$

and so

$$
\left\|p_{h}-p^{*}\right\| \leq C\left(\left\|u_{h}-u^{*}\right\|_{V}+\left\|u-u^{*}\right\|_{V}+\left\|\left|p-p^{*}\right|\right\|\right) .
$$

Combining these estimates, we get

$$
\begin{aligned}
& \left\|u_{h}-u^{*}\right\|_{V}+\left\|p_{h}-p^{*}\right\| \mid+\epsilon\left\|p_{h}-p^{*}\right\|_{W} \\
& \quad \leq C\left(\left\|u-u^{*}\right\|_{V}+\left\|p-p^{*}\right\| \mid+\epsilon\left\|p-p^{*}\right\|_{W}\right),
\end{aligned}
$$

from which the theorem follows. 


\section{Finite Elements for the NAGHDi Shell}

Our goal is now to discretize the mixed formulation of the Naghdi shell model presented in $\S 2$ and analyze the error in the framework of $\S 3$. We take the spaces $V$ and $W$ and the forms $A, B, C$, and $F$ as defined in $\S 2$ and set $\epsilon=t / \sqrt{1-c_{0} t^{2}}=$ $O(t)$. Clearly, the forms are all bounded and $C$ is positive definite. In light of Lemma $1, A$ is positive definite as well. Hence, Theorem 2 gives the well-posedness of the mixed formulation, and for any choice of finite element spaces $V_{h} \subset V$ and $W_{h} \subset W$, Theorem 3 gives a convergence result. However, we wish to obtain a convergence estimate which is uniform in $t$, and so want to apply Theorem 4 . This requires that we find finite element spaces satisfying (16) with $\gamma_{h}$ bounded below by a positive constant independent of $h$.

To do so, we henceforth assume that $\Omega$ is a polygon which is triangulated by a regular sequence of triangulations $\mathcal{T}_{h}$ indexed by the meshsize $h$. (By regular, we mean that there is a positive lower bound for all the angles of all the triangles in all the triangulations, but we do not assume quasi-uniformity.) The set $\Gamma_{d}$ where Dirichlet boundary conditions are imposed is assumed to be a union of edges of triangles in each $\mathcal{T}_{h}$. We use the notation $\mathcal{P}_{k}(T)$ for the set of functions on $T$ which are the restrictions of polynomials of degree no greater than $k$ and define

$$
\begin{aligned}
& L_{k}^{s}\left(\mathcal{T}_{h}\right)=\left\{v \in H^{s}(\Omega)|v|_{T} \in \mathcal{P}_{k}(T) \text { for all } T \in \mathcal{T}_{h}\right\}, \\
& B_{k}\left(\mathcal{T}_{h}\right)=\left\{v \in L_{k}^{0}\left(\mathcal{T}_{h}\right) \mid v \in \stackrel{H}{H}^{1}(T) \text { for all } T \in \mathcal{T}_{h}\right\} .
\end{aligned}
$$

For the shell problem we shall take

$$
\begin{aligned}
V_{h} & =\left\{(\vec{v}, \underset{\sim}{\psi}) \in V \mid v_{i}, \psi_{\alpha} \in L_{k+2}^{1}\left(\mathcal{T}_{h}\right)+B_{k+3}\left(\mathcal{T}_{h}\right)\right\}, \\
W_{h} & =\left\{(\underset{\sim}{\sim} \underset{\sim}{\chi}) \in W \mid \eta_{\alpha}, \chi_{\alpha \beta} \in L_{k}^{0}\left(\mathcal{T}_{h}\right)\right\},
\end{aligned}
$$

where $k$ is any fixed nonnegative integer (this method shall turn out to converge with order $k+1$ in $H^{1}$ for displacement and rotation). That is, we use Lagrange finite elements of degree $k+2$ augmented by bubble functions of one degree higher for the components of the displacement and rotation, and discontinuous piecewise polynomials of degree $k$ for the components of the shear and membrane stresses. The nodal diagrams for this element choice are shown in Figure 1.

Before proceeding to the analysis, we discuss briefly the implementation of this method. The stress unknowns may be eliminated analytically from the discrete equations, giving a finite element method involving only the displacements and

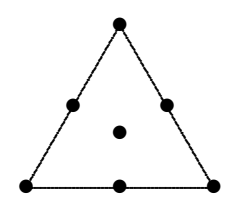

$u_{i}, \theta_{\alpha}$

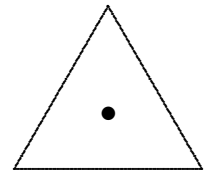

$\zeta_{\alpha}, \lambda_{\alpha \beta}$

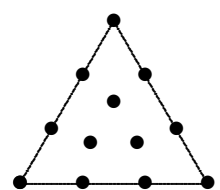

$u_{i}, \theta_{\alpha}$

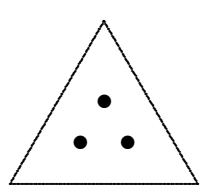

$\zeta_{\alpha}, \lambda_{\alpha \beta}$

FiguRE 1. Degrees of freedom for displacements and rotations and for shear stresses and membrane stresses in the cases $k=0$ and $k=1$ 
rotations. Specifically, let

$$
P_{h}^{1}:\left\{\underset{\sim}{\eta} \mid \eta_{\alpha} \in L^{2}(\Omega)\right\} \rightarrow\left\{\underset{\sim}{\eta} \mid \eta_{\alpha} \in L_{k}^{0}\left(\mathcal{T}_{h}\right)\right\}
$$

denote the orthogonal projection with respect to the inner product

$$
(\underset{\sim}{\phi}, \underset{\sim}{\eta}) \mapsto \int_{\Omega} a^{\alpha \beta} \phi_{\alpha} \eta_{\beta} \sqrt{a} d x
$$

and, similarly, let

$$
P_{h}^{2}:\left\{\underset{\approx}{\chi} \mid \chi_{\alpha \beta} \in L^{2}(\Omega)\right\} \rightarrow\left\{\underset{\approx}{\chi} \mid \chi_{\alpha \beta} \in L_{k}^{0}\left(\mathcal{T}_{h}\right)\right\}
$$

denote the orthogonal projection with respect to the inner product

$$
(\underset{\approx}{\underset{\approx}{\chi} \underset{\approx}{\chi}}) \mapsto \int_{\Omega} a^{\alpha \gamma} a^{\beta \delta} \lambda_{\alpha \beta} \chi_{\beta \delta} \sqrt{a} d x
$$

From the discrete version of $(8)$ we deduce that

$$
\underset{\sim}{\phi}=\left(t^{-2}-c_{0}\right) \frac{E}{2(1+\nu)} P_{h}^{1} \underset{\sim}{\Phi}\left(\vec{u}_{h}, \underset{\sim}{\theta}\right), \quad \underset{\approx_{h}}{\lambda}=\left(t^{-2}-c_{0}\right) P_{h}^{2} \Lambda_{\sim}^{*}\left(\vec{u}_{h}\right),
$$

where

$$
\Lambda_{\gamma \delta}^{*}\left(\vec{u}_{h}\right)=\frac{E}{1-\nu^{2}}\left[\Lambda_{\gamma \delta}\left(\vec{u}_{h}\right)+\nu a_{\gamma \delta} a^{\alpha \beta} \Lambda_{\alpha \beta}\left(\vec{u}_{h}\right)\right]
$$

It then follows from the discrete version of $(7)$ that $\left(\vec{u}_{h}, \underset{\sim}{\theta}\right)$ can be determined as the minimizer over $V_{h}$ of the altered energy functional

$$
E_{h}(\vec{v}, \underset{\sim}{\psi})=E^{B}+c_{0} t^{2} E^{S}+\left(1-c_{0} t^{2}\right) E_{h}^{S}+c_{0} t^{2} E^{M}+\left(1-c_{0} t^{2}\right) E_{h}^{M}+E^{L},
$$

where $E^{B}, E^{S}, E^{M}$, and $E^{L}$ are defined in (5) and $E_{h}^{S}$ and $E_{h}^{M}$ are derived from $E^{S}$ and $E^{M}$ by replacing $\underset{\sim}{\phi}$ and $\underset{\approx}{\Lambda}$ by $P_{h}^{1} \underset{\sim}{\phi}$ and $P_{h}^{2} \underset{\approx}{\Lambda}$, respectively. The insertion of such projections and other reduction operators into energy terms subject to locking is known in the engineering literature as "selective reduced integration." Since we introduce the projections into only part of the shear and membrane energy terms, our method may be described as "partial selective reduced integration."

We now turn to the major task of this paper, the verification of (16) for the elements just introduced. Unfortunately, in order to do so, we need to make an assumption on the coefficients entering into the bilinear form $B$. Namely, we assume that each of the quantities $a_{\alpha \beta}, b_{\alpha \beta}$, and $\Gamma_{\alpha \beta}^{\gamma}$ belong to $L_{0}^{0}\left(\mathcal{T}_{h}\right)$, that is, they are piecewise constant. Of course this assumption is very restrictive. It is introduced only because it is necessary for the numerical analysis. We conjecture that this restriction is a consequence of the technique of proof, and that in fact it is unnecessary. With this restriction, we are able to prove our main result.

Theorem 5. Assume that $a^{\alpha \beta}, b_{\alpha \beta}, \Gamma_{\alpha \beta}^{\delta}$ belong to $L_{0}^{0}\left(\mathcal{T}_{h}\right)$, and let a denote the determinant of $\left(a_{\alpha \beta}\right),\left(a^{\alpha \beta}\right)$ the inverse matrix, and $b_{\alpha}^{\gamma}=a^{\gamma \beta} b_{\alpha \beta}$. Define forms $A, B, C$, and $F$ by (1)-(4) and (9)-(12), where $f^{i} \in L^{2}(\Omega), E>0, \nu \in(0,1 / 2)$, $c_{0}>0$, and $t \in\left(0,1 / \sqrt{c_{0}}\right)$. Then there exist unique pairs $(\vec{u} \underset{\sim}{\theta}) \in V$ and $\left.\underset{\sim}{\phi} \underset{\sim}{\lambda}\right) \in W$ satisfying $(7)$ and $(8)$, and unique pairs $\left(\vec{u}_{h}, \underset{\sim_{h}}{\theta}\right) \in V_{h}$ and $\left(\underset{\sim_{h}}{\phi}, \underset{\approx_{h}}{\lambda}\right) \in W_{h}$ satisfying 
the same equations with the test functions restricted to $V_{h}$ and $W_{h}$. Moreover, there exists a constant $C$ independent of $h, t$, and $\vec{f}$ such that

$$
\begin{aligned}
& \left\|\vec{u}-\vec{u}_{h}\right\|_{1}+\|\underset{\sim}{\theta}-\underset{\sim}{\theta}\|_{1}+\|\underset{\sim}{\phi}-\underset{\sim}{\phi}, \underset{\approx}{\lambda}-\underset{\approx}{\lambda}\|\|+t\| \underset{\sim}{\phi}-\underset{\sim}{\phi}\left\|_{0}+t\right\| \underset{\approx}{\lambda}-\underset{\approx}{\lambda} \|_{0} \\
& \leq C \inf _{(\vec{v}, \psi) \in V_{h}}\left(\|\vec{u}-\vec{v}\|_{1}+\|\underset{\sim}{\theta}-\underset{\sim}{\psi}\|_{1}+\|\underset{\sim}{\phi}-\underset{\sim}{\eta} \underset{\approx}{\lambda}-\underset{\approx}{\chi}\|\|+t\| \underset{\sim}{\phi}-\underset{\sim}{\eta}\left\|_{0}+t\right\| \underset{\approx}{\underset{\approx}{\sim}}-\underset{\approx}{\chi \|_{0}}\right), \\
& (\underset{\sim}{\sim}, \underset{\sim}{\mathcal{X}}) \in W_{h}
\end{aligned}
$$

where

$$
\|\underset{\sim}{\eta}, \underset{\sim}{\chi}\|:=\sup _{(\vec{v}, \underset{\sim}{\psi}) \in V} \frac{B(\vec{v}, \underset{\sim}{\psi} ; \underset{\sim}{\eta}, \underset{\sim}{\chi})}{\|\vec{v}, \underset{\sim}{\psi}\|_{V}} .
$$

Since $\|\underset{\sim}{\eta} \underset{\sim}{\chi} \underset{\sim}{\chi}\| \leq\left(\|\underset{\sim}{\eta}\|_{0}+\|\underset{\sim}{\chi}\|_{0}\right)$ we immediately get $k$ th-order convergence uniform in $t$ for smooth solutions.

Corollary 6. Under the hypotheses of the theorem we have

$$
\begin{aligned}
\left\|\vec{u}-\vec{u}_{h}\right\|_{1}+\|\underset{\sim}{\theta}-\underset{\sim}{\theta}\|_{h}+\||\underset{\sim}{\phi}-\underset{\sim}{\phi}, \underset{\approx}{\lambda}-\underset{\approx}{\lambda}|\|+t\|\underset{\sim}{\phi}-\underset{\sim}{\phi}\|_{0}+t\|\underset{\approx}{\lambda}-\underset{\approx}{\lambda}\|_{0} \\
\leq C h^{k+1}\left(\|\vec{u}\|_{k+2}+\|\underset{\sim}{\theta}\|_{k+2}+\|\underset{\sim}{\phi}\|_{k+1}+\| \underset{\approx}{\lambda_{k+1}}\right),
\end{aligned}
$$

where the constant $C$ does not depend on $h, t$, or $\vec{f}$.

Before turning to the proof of Theorem 5 and Corollary 6 , we discuss their interpretation. Let $U$ denote the solution $(\vec{u}, \underset{\sim}{\theta} \underset{\sim}{\phi} \underset{\sim}{\lambda})$ and set

$$
|U|=\|\vec{u}\|_{1}+\|\underset{\sim}{\theta}\|_{1}+\|\underset{\sim}{\phi} \underset{\approx}{\lambda}\| \mid+t\|\underset{\sim}{\phi}\|_{0}+t\|\underset{\approx}{\lambda}\|_{0} .
$$

Similarly, define $U_{h}$ to be the discrete solution. Then $|U|$ is bounded above and below by positive constants uniformly in $t$, and, assuming that the solution is smooth enough that the norms on the right-hand side of (17) remain bounded uniformly in $t$, the error $\left|U-U_{h}\right|$ is uniformly of the order $O\left(h^{k+1}\right)$. In particular, the relative error $\left|U-U_{h}\right| /|U|$ tends to zero as fast as is permitted by the approximation properties of the finite element spaces used, that is with $O\left(h^{k+1}\right)$, uniformly in $t$. In this sense we have indeed shown our method to be free of locking. However, this statement may be misleading, so we comment on several aspects of it.

First, from the point of view of approximation order, our choice of elements is unbalanced. The finite element space for displacement and rotation contains all Lagrange elements of degree $k+2$, and so is capable of approximating the displacements and rotations with order $k+2$ in $H^{1}$, but, because of the lower order of approximation of the elements used for the stress fields, only convergence of order $k+1$ is obtained. In fact, the shape functions of degree greater than $k+1$ for displacement and rotation are used to achieve stability rather than accurate approximation. An analogous situation is the use of quadratic shape functions for velocity in the $P_{2}-P_{0}$ Stokes element. A careful reading of the analysis below shows that we can reduce the space used for the rotation variables to $L_{k+1}^{1}\left(\mathcal{T}_{h}\right)+B_{k+2}\left(\mathcal{T}_{h}\right)$ and still retain stability, but this is not possible for the displacement variables. Actually, our choice of elements was made primarily for the relative simplicity of the stability analysis which follows. It is likely that with a more sophisticated analysis, for example one employing macroelement techniques, better balanced and 
more economical elements could be shown to be stable for the mixed formulation of this paper.

Second, while the norm $\|\cdot \mid\|$ in which we measure the stresses arises naturally from the shell problem, it is not easy to interpret it concretely, and its exact relationship to Sobolev norms depends on the geometry of the shell and the boundary conditions. Thus, the significance of the $t$-independent bound on the error in the stress variables is unclear.

Third, while it cannot happen under our scaling that $|U|$ tends to zero with $t$, it may well happen that $\|\vec{u}\|_{1}+\|\underset{\sim}{\sim}\|_{1}$ tends to zero. This is the case of a membranedominated shell problem. In this case our results, which couple the displacement and stress errors, do not establish convergence of the displacement-rotation relative error

$$
\frac{\left\|u-\vec{u}_{h}\right\|_{1}+\|\underset{\sim}{\theta}-\underset{\sim}{\theta}\|_{h}}{\|u\|_{1}+\|\underset{\sim}{\theta}\|_{1}}
$$

uniformly in $t$. However, locking is generally considered a problem mostly in the case of bending-dominated shell problems, i.e., problems for which $\|\vec{u}\|_{1}$ remains bounded away from zero as $t$ tends to zero, and our results do imply uniform convergence rates for the displacement and rotation in that case.

Fourth, the right-hand side of (17) cannot be expected to remain bounded as $t$ tends to zero. The analogous quantity for the Reissner-Mindlin plate model is known to become unbounded, owing to boundary layers, as has been analyzed thoroughly in [3]. No such analysis yet exists for the Naghdi shell model. This lack of uniform regularity of the solution will preclude uniform $O\left(h^{k+1}\right)$ convergence of the numerical method except under special conditions (e.g., periodic problems). This difficulty, however, is quite distinct from the locking problem treated in this paper. In any case, Theorem 5 shows that the finite element solution provides the best approximation that the regularity of the solution allows, uniformly with respect to $t$. Moreover, when the presence of boundary layers precludes the optimal rates of convergence uniform in $t$, it is still possible that such convergence occurs on interior subdomains. Interior convergence results of this sort have been established for some Reissner-Mindlin plate elements in [10] and [13]. We also mention that by analyzing more carefully the norm $\|\cdot \mid\|$, instead of simply bounding it by the $L^{2}$ norm, the norm on the solution which appears on the right-hand side of (17) can probably be replaced with a weaker one (but generally not by one that is bounded uniformly in $t$ ). Cf. the use of the Helmholtz Theorem to treat the analogous term in the Reissner-Mindlin plate model [2].

We now turn to the proof of Theorem 5 . In view of Theorem 4, it suffices to establish the following lemma.

Lemma 7. Under the hypotheses of the theorem there exists a positive number $\gamma$ independent of $h$ for which

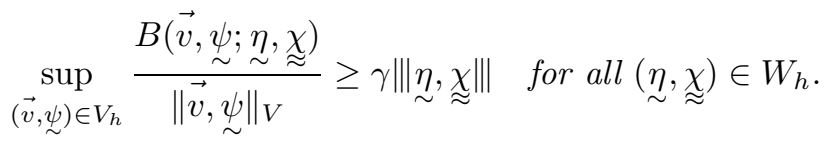

Proof. Suppose that we can construct a linear operator

$$
\pi_{h}: H_{\Gamma_{d}}^{1} \rightarrow L_{k+2}^{1}\left(\mathcal{T}_{h}\right) \cap H_{\Gamma_{d}}^{1}+B_{k+3}\left(\mathcal{T}_{h}\right)
$$


for which

$$
\left\|\pi_{h} v\right\|_{1} \leq C\|v\|_{1} \quad \text { for all } v \in H_{\Gamma_{d}}^{1},
$$

with $C$ independent of $h$ and such that

$$
\begin{aligned}
\int_{e}(v & \left.-\pi_{h} v\right) p d s \\
& =\int_{T}\left(v-\pi_{h} v\right) p \underset{\sim}{d x}=0 \quad \text { for all } e \in \mathcal{E}_{h}, T \in \mathcal{T}_{h}, v \in H_{\Gamma_{d}}^{1}, p \in \mathcal{P}_{k}(T),
\end{aligned}
$$

where $\mathcal{E}_{h}$ denotes the set of all edges of triangles in $\mathcal{T}_{h}$. Note that it follows from Green's theorem and (21) that

$$
\int_{T}\left(v-\pi_{h} v\right)_{, \alpha} p d x=0 \quad \text { for all } T \in \mathcal{T}_{h}, v \in H_{\Gamma_{d}}^{1}, p \in \mathcal{P}_{k}(T),
$$

as well. Therefore, if we define $\Pi_{h}: V \rightarrow V_{h}$ by $\Pi_{h}(\vec{v}, \underset{\sim}{\psi})=\left(\left(\pi_{h} v_{i}\right),\left(\pi_{h} \psi_{\alpha}\right)\right)$, we have

$$
\left.B\left(\Pi_{h}(\vec{v}, \underset{\sim}{\psi}) ; \underset{\sim}{\eta}, \underset{\approx}{\chi}\right)=B(\vec{v}, \underset{\sim}{\psi} \underset{\sim}{\eta}, \underset{\approx}{\chi}) \quad \text { for all } \underset{\sim}{\sim}, \underset{\approx}{\chi}\right) \in W_{h}
$$

and

$$
\left\|\Pi_{h}(\vec{v}, \underset{\sim}{\psi})\right\|_{V} \leq C_{0}\|\vec{v}, \underset{\sim}{\psi}\|_{V}
$$

with $C_{0}$ independent of $h$. Then, given $\left.\underset{\sim}{\eta}, \underset{\approx}{\chi}\right) \in W_{h}$, we may choose $(\vec{v}, \underset{\sim}{\psi}) \in V$ for which

$$
\frac{B(\vec{v}, \underset{\sim}{\psi} ; \underset{\sim}{\eta} \underset{\approx}{\underset{\sim}{\chi}})}{\|\vec{v}, \underset{\sim}{\psi}\|_{V}} \geq \frac{1}{2}\|\underset{\sim}{\eta} \underset{\approx}{\chi}\|
$$

(which is possible by the definition of the seminorm) and bound the left-hand side of (18) below by

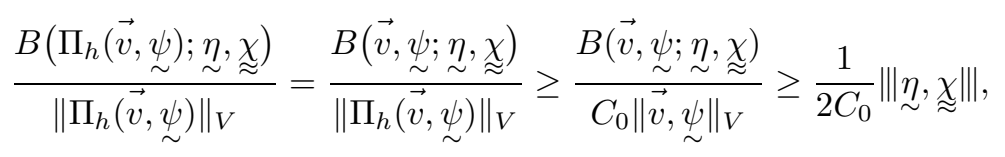

so the lemma holds with $\gamma=1 /\left(2 C_{0}\right)$.

It remains to construct the operator $\pi$ satisfying (19)-(21), which we do in four steps. First, let $\pi_{h}^{0}: H_{\Gamma_{d}}^{1} \rightarrow L_{1}^{1}\left(\mathcal{T}_{h}\right) \cap H_{\Gamma_{d}}^{1}$ satisfy

$$
\left\|v-\pi_{h}^{0} v\right\|_{0, T}+h_{T}\left\|v-\pi_{h}^{0} v\right\|_{1, T} \leq C h_{T}\|v\|_{1, \tilde{T}} \quad \text { for all } T \in \mathcal{T}_{h}, v \in H_{\Gamma_{d}}^{1} .
$$

Here, $h_{T}$ denotes the diameter of the triangle $T$, and $\tilde{T}$ denotes the union of the triangles in $\mathcal{T}_{h}$ which meet $T$. The construction of such an operator is described in [9]. Second, define

$$
\pi_{h}^{1}: H_{\Gamma_{d}}^{1} \rightarrow L_{k+2}^{1}\left(\mathcal{T}_{h}\right) \cap H_{\Gamma_{d}}^{1}
$$

by the conditions $\pi_{h}^{1} v=0$ at all vertices of all triangles in $\mathcal{T}_{h}$ and

$$
\begin{array}{ll}
\int_{e}\left(v-\pi_{h}^{1} v\right) p d s=0 & \text { for all } e \in \mathcal{E}_{h}, v \in H_{\Gamma_{d}}^{1}, p \in \mathcal{P}_{k}(e), \\
\int_{T}\left(v-\pi_{h}^{1} v\right) p d \underset{\sim}{d x}=0 & \text { for all } T \in \mathcal{T}_{h}, v \in H_{\Gamma_{d}}^{1}, p \in \mathcal{P}_{k-1}(T) .
\end{array}
$$


By a straightforward scaling argument we obtain

$\left\|v-\pi_{h}^{1} v\right\|_{0, T}+h_{T}\left\|v-\pi_{h}^{1} v\right\|_{1, T} \leq C\left(\|v\|_{0, T}+h_{T}\|v\|_{1, T}\right) \quad$ for all $T \in \mathcal{T}_{h}, v \in H_{\Gamma_{d}}^{1}$.

Third, define $\pi_{h}^{2}: H_{\Gamma_{d}}^{1} \rightarrow B_{k+3}\left(\mathcal{T}_{h}\right)$ by

$$
\int_{T}\left(v-\pi_{h}^{2} v\right) p \underset{\sim}{\sim}=0 \quad \text { for all } T \in \mathcal{T}_{h}, v \in H_{\Gamma_{d}}^{1}, p \in \mathcal{P}_{k}(T) .
$$

For this operator, scaling gives

$$
\left\|v-\pi_{h}^{2} v\right\|_{0, T}+h_{T}\left\|v-\pi_{h}^{2} v\right\|_{1, T} \leq C\|v\|_{0, T} \quad \text { for all } T \in \mathcal{T}_{h}, v \in H_{\Gamma_{d}}^{1} .
$$

Finally, we set

$$
\pi_{h} v=\pi_{h}^{0} v+\pi_{h}^{1}\left(v-\pi_{h}^{0} v\right)+\pi_{h}^{2}\left[v-\pi_{h}^{0} v-\pi_{h}^{1}\left(v-\pi_{h}^{0} v\right)\right] .
$$

From (22), (24), and (26) we deduce that

$$
\left\|\pi_{h} v\right\|_{1, T} \leq C\|v\|_{1, \tilde{T}} \quad \text { for all } v \in H_{\Gamma_{d}}^{1}, T \in \mathcal{T}_{h},
$$

and (21) follows immediately, while (20) follows from (23), (25), and (27). This completes the proof of Lemma 7 , and so of Theorem 5 .

\section{REFERENCES}

1. D. N. Arnold and F. Brezzi, Some new elements for the Reissner-Mindlin plate model, Boundary Value Problems for Partial Differential Equations and Applications (C. Baiocchi and J.-L. Lions, eds.), Masson, Paris, 1993, pp. 287-292. MR 94k:73066

2. D. N. Arnold and R. S. Falk, A uniformly accurate finite element method for the ReissnerMindlin plate, SIAM J. Numer. Anal. 26 (1989), 1276-1290. MR 91c:65068

3. _ Asymptotic analysis of the boundary layer for the Reissner-Mindlin plate model, SIAM J. Math. Anal. 27 (1996), 486-514. CMP 96:09

4. I. Babuška and M. Suri, On locking and robustness in the finite element method, SIAM J. Numer. Anal. 29 (1992), 1261-1293. MR 94c:65128

5. K.-J. Bathe and E. Wilson, Numerical methods in finite element analysis, Prentice-Hall, Englewood Cliffs, NJ, 1976.

6. M. Bernadou, P. G. Ciarlet, and B. Miara, Existence theorems for two-dimensional linear shell theories, J. Elasticity 34 (1992), 111-138. MR 95f:73050

7. F. Brezzi and M. Fortin, Mixed and hybrid finite element methods, Springer-Verlag, New York-Heidelberg-Berlin, 1991. MR 92d:65187

8. F. Brezzi, M. Fortin, and D. Marini, Mixed finite element methods with continuous stresses, Math. Mod. Methods Appl. Sci. 3 (1993), 275-287. MR 94g:65121

9. P. Clement, Approximation by finite element functions using local regularization, RAIRO, Anal. Numér. 9 (1975), 33-76. MR 53:4569

10. L. Gastaldi, Uniform interior error estimates for the Reissner-Mindlin plate model, Math. Comp. 61 (1993), 539-568. MR 94a:65067

11. V. Girault and P. A. Raviart, Finite element methods for Navier-Stokes equations, SpringerVerlag, New York-Heidelberg-Berlin, 1986. MR 88b:65129

12. T. J. R. Hughes, The finite element method, linear static and dynamic finite element analysis, Prentice-Hall, Englewood Cliffs, NJ, 1987. MR 90i:65001

13. X. Liu, Interior estimates for some mixed finite element methods, Ph.D. thesis, Penn State Univ., 1993.

14. P. M. Naghdi, Foundations of elastic shell theory, Progress in Solid Mechanics, Vol. 4 (I. N. Sneddon and R. Hill, eds.), North-Holland, Amsterdam, 1963, pp. 1-90. MR 29:790

15. P. M. Naghdi, The theory of shells and plates, Handbuch der Physik, Vol. VI a-2, SpringerVerlag, New York-Heidelberg-Berlin, 1972, pp. 425-640.

16. J. Pitkäranta, The problem of membrane locking in finite element analysis of cylindrical shells, Numer. Math. 61 (1992), 523-542. MR 93b:65178 
17. T.-X. Zhou, The partial projection method in the finite element discretization of the ReissnerMindlin plate, J. Comput. Math 13 (1995), 172-191. CMP 95:12

18. O. C. Zienkiewicz and R. L. Taylor, The finite element method, McGraw-Hill, New York, NY, 1989. MR 96c:73069

Department of Mathematics, The Pennsylvania State University, University Park, Pennsylvania 16802

E-mail address: dna@math.psu.edu

Istituto di Analisi Numerica del C.N.R., Università di Pavia, 27100 Pavia, Italy

E-mail address: brezzi@dragon.ian.pv.cnr.it 